\title{
MEAN GROWTH OF THE DERIVATIVE OF ANALYTIC FUNCTIONS, BOUNDED MEAN OSCILLATION AND NORMAL FUNCTIONS
}

\author{
Oscar Blasco, Daniel Girela and M. Auxiliadora Márquez
}

\begin{abstract}
For a given positive function $\phi$ defined in $[0,1)$ and $1 \leq p<\infty$, we consider the space $\mathcal{L}(p, \phi)$ which consists of all functions $f$ analytic in the unit disc $\Delta$ for which $\left(\frac{1}{2 \pi} \int_{-\pi}^{\pi}\left|f^{\prime}\left(r e^{i \theta}\right)\right|^{p} d \theta\right)^{1 / p}=\mathrm{O}(\phi(r))$, as $r \rightarrow 1$. A result of Bourdon, Shapiro and Sledd implies that such a space is contained in $B M O A$ for $\phi(r)=(1-r)^{\frac{1}{p}-1}$. Among other results, in this paper we prove that this result is sharp in a very strong sense, showing that, for a large class of weight functions $\phi$, the function $\phi(r)=(1-r)^{\frac{1}{p}-1}$ is the best one to get $\mathcal{L}(p, \phi) \subset B M O A$. Actually, if $\phi(r)(1-r)^{1-\frac{1}{p}} \uparrow \infty$, as $r \uparrow 1$, we construct a function $f \in \mathcal{L}(p, \phi)$ which is not a normal function. These results improve other obtained recently by the second author. We also characterize the functions $\phi$, among a certain class of weight functions, to be able to embedd $\mathcal{L}(p, \phi)$ into $H^{q}$ for $q>p$ or into the space $\mathcal{B}$ of Bloch functions.
\end{abstract}

\section{INTRODUCTION AND STATEMENT OF RESULTS.}

Let $\Delta$ denote the unit disc $\{z \in \mathbb{C}:|z|<1\}$ and $\mathbb{T}$ the unit circle $\{\xi \in \mathbb{C}:|\xi|=1\}$. If $0<r<1$ and $g$ is a function which is analytic in $\Delta$, we set

$$
\begin{gathered}
M_{p}(r, g)=\left(\frac{1}{2 \pi} \int_{-\pi}^{\pi}\left|g\left(r e^{i \theta}\right)\right|^{p} d \theta\right)^{1 / p}, \quad 0<p<\infty, \\
M_{\infty}(r, g)=\max _{|z|=r}|g(z)| .
\end{gathered}
$$

For $0<p \leq \infty$ the Hardy space $H^{p}$ consists of those functions $g$, analytic in $\Delta$, for which

$$
\|g\|_{H^{p}}=\sup _{0<r<1} M_{p}(r, g)<\infty .
$$

The space $B M O A$ consists of those functions $f \in H^{1}$ whose boundary values have bounded mean oscillation on $\mathbb{T}$. We refer to [3] and [9] for the main properties of BMOAfunctions.

1980 Mathematics Subject Classification (1985 Revision). Primary 30D55, 30D45. Secondary $30 \mathrm{C} 45$.

The research of the first author has been supported in part by a D. G. I. C. Y. T. grant (PB95-0261) while that of the second and third authors has been supported in part by a D. G. I. C. Y. T. grant (PB94-1496) and by a grant from "La Junta de Andalucía". . 
In this paper we shall be dealing with functions $f$, analytic in $\Delta$, for which the integral means $M_{p}\left(r, f^{\prime}\right)$ are dominated by a certain weight function $\phi(r)$. Namely, let $1 \leq p \leq \infty$ and let $\phi$ be a non-negative function defined in $[0,1)$. We define the space $\mathcal{L}(p, \phi)$ as the space of all functions $f$ analytic in $\Delta$ for which

$$
M_{p}\left(r, f^{\prime}\right)=\mathrm{O}(\phi(r)), \quad \text { as } r \rightarrow 1 .
$$

The spaces $\mathcal{L}(p, \phi)$ are closely related to mean Lipschitz spaces. If $f$ is a function which is analytic in $\Delta$ and has a non-tangential limit $f\left(e^{i \theta}\right)$ at almost every $e^{i \theta} \in \mathbb{T}$, we define

$$
\begin{aligned}
& \omega_{p}(\delta, f)=\sup _{0<|t| \leq \delta}\left(\frac{1}{2 \pi} \int_{-\pi}^{\pi}\left|f\left(e^{i(\theta+t)}\right)-f\left(e^{i \theta}\right)\right|^{p} d \theta\right)^{1 / p}, \quad \delta>0, \quad \text { if } 1 \leq p<\infty, \\
& \omega_{\infty}(\delta, f)=\sup _{0<|t| \leq \delta}\left(\operatorname{ess.sup}_{\theta \in[-\pi, \pi]}\left|f\left(e^{i(\theta+t)}\right)-f\left(e^{i \theta}\right)\right|\right), \quad \delta>0 .
\end{aligned}
$$

Then $\omega_{p}(., f)$ is the integral modulus of continuity of order $p$ of the boundary values $f\left(e^{i \theta}\right)$ of $f$.

Throughout the paper $\omega:[0,1] \rightarrow[0, \infty)$ will be a continuous and increasing function with $\omega(0)=0$. Then, for $1 \leq p \leq \infty$, the mean Lipschitz space $\Lambda(p, \omega)$ consists of those functions $f \in H^{p}$ which satisfy

$$
\omega_{p}(\delta, f)=\mathrm{O}(\omega(\delta)), \quad \text { as } \delta \rightarrow 0 .
$$

If $0<\alpha \leq 1$ and $\omega(\delta)=\delta^{\alpha}$, we shall write $\Lambda_{\alpha}^{p}$ instead of $\Lambda(p, \omega)$, that is, we set

$$
\Lambda_{\alpha}^{p}=\Lambda\left(p, \delta^{\alpha}\right), \quad 0<\alpha \leq 1, \quad 1 \leq p \leq \infty
$$

A classical result of Hardy and Littlewood [12] (see also Chapter 5 of [7]) asserts that for $1 \leq p \leq \infty$ and $0<\alpha \leq 1$, we have that

$$
\Lambda_{\alpha}^{p}=\left\{f \text { analytic in } \Delta: M_{p}\left(r, f^{\prime}\right)=\mathrm{O}\left(\frac{1}{(1-r)^{1-\alpha}}\right), \quad \text { as } r \rightarrow 1\right\} .
$$

Notice that $(1.2)$ can be written as

$$
\Lambda_{\alpha}^{p}=\mathcal{L}\left(p, \frac{1}{(1-r)^{1-\alpha}}\right), \quad 1 \leq p \leq \infty, \quad 0<\alpha \leq 1 .
$$

The question of finding conditions on $\omega$ so that it is possible to obtain results on the spaces $\Lambda(p, \omega)$ analogous to those proved by Hardy and Littlewood for the spaces $\Lambda_{\alpha}^{p}$ has been studied by several authors (see e.g. [4] and [16]). We shall say that $\omega$ satisfies the Dini condition or that $\omega$ is a Dini-weight if there exists a positive constant $C$ such that

$$
\int_{0}^{\delta} \frac{\omega(t)}{t} d t \leq C \omega(\delta), \quad 0<\delta<1 .
$$

Given $0<q<\infty$, we shall say that $\omega$ satisfies the condition $b_{q}$ or that $\omega \in b_{q}$ if there exists a positive constant $C$ such that

$$
\int_{\delta}^{1} \frac{\omega(t)}{t^{q+1}} d t \leq C \frac{\omega(\delta)}{\delta^{q}}, \quad 0<\delta<1 .
$$

The infimum over all possible constants verifying (1.5) will be denoted by $\|\omega\|_{b_{q}}$.

The first author and de Souza proved in [4, Th. 2.1] the following extension of (1.2). 
Theorem A. Let $1 \leq p \leq \infty$ and let $\omega:[0,1] \rightarrow[0, \infty)$ be a continuous and increasing function with $\omega(0)=0$. If $\omega$ is a Dini-weight and satisfies the condition $b_{1}$ then,

$$
\Lambda(p, \omega)=\left\{f \text { analytic in } \Delta: M_{p}\left(r, f^{\prime}\right)=\mathrm{O}\left(\frac{\omega(1-r)}{1-r}\right), \text { as } r \rightarrow 1\right\},
$$

or, equivalently,

$$
\Lambda(p, \omega)=\mathcal{L}\left(p, \frac{\omega(1-r)}{1-r}\right) .
$$

A well known result of Privalov [7, Th. 3.11] asserts that a function $f$ analytic in $\Delta$ has a continuous extension to the closed unit disc $\bar{\Delta}$ whose boundary values are absolutely continuous on $\mathbb{T}$ if and only if $f^{\prime} \in H^{1}$. Consequently, using (1.2), we can state the following.

Theorem B. Let $f$ be a function which is analytic in $\Delta$. Then, the three following conditions are equivalent:

(i) $f \in \Lambda_{1}^{1}$.

(ii) $f^{\prime} \in H^{1}$.

(iii) $f$ has a continuous extension to the closed unit disc $\bar{\Delta}$ whose boundary values are absolutely continuous on $\mathbb{T}$.

Cima and Petersen proved in [6] that $\Lambda_{1 / 2}^{2} \subset B M O A$. This result was extended by Bourdon, Shapiro and Sledd who proved the following result in [5].

Theorem C. For $1<p<\infty, \Lambda_{1 / p}^{p} \subset B M O A$.

The second author has recently proved in [10] and [11] that Theorem B and Theorem $\mathrm{C}$ are sharp in a very strong sense. In this paper we shall improve these results.

We recall that a function $f$ analytic in $\Delta$ is a Bloch function if

$$
\sup _{z \in \Delta}\left(1-|z|^{2}\right)\left|f^{\prime}(z)\right|<\infty .
$$

The space of all Bloch functions is denoted by $\mathcal{B}$. Notice that we have $\mathcal{B}=\mathcal{L}\left(\infty, \frac{1}{1-r}\right)$. It is well known that

$$
H^{\infty} \subset B M O A \subset \mathcal{B}
$$

Note that $\omega(t)=t^{\alpha}$ for $0<\alpha<1$ is a Dini and $b_{1}$ weight. We can state the following result, which says that Theorem $\mathrm{C}$ is the best among these weights.

Theorem 1.1. Let $1<p<\infty$ and let $\omega$ be a Dini-weight, continuous, non-decreasing with $\omega(0)=0$ and $\omega \in b_{1}$. Then the following conditions are equivalent:

(i) $\Lambda(p, \omega) \subset B M O A$.

(ii) $\Lambda(p, \omega) \subset \mathcal{B}$.

(iii) $\omega(\delta)=\mathrm{O}\left(\delta^{1 / p}\right), \quad$ as $\delta \rightarrow 0$.

In Theorem 1.1 the implication (i) $\Rightarrow$ (ii) follows trivially from the inclusion $B M O A \subset$ $\mathcal{B}$ and the implication (iii) $\Rightarrow$ (i) follows from Theorem $\mathrm{C}$. The remaining implication, (ii) $\Rightarrow$ (iii), follows from the following result. 
Theorem 1.2. Let $1<p<\infty$ and let $\omega:[0,1) \rightarrow[0, \infty)$ be a continuous and nondecreasing function with $\omega(0)=0$ such that $\omega$ is a Dini-weight and $\omega \in b_{1}$. If

$$
\limsup _{\delta \rightarrow 0} \frac{\omega(\delta)}{\delta^{1 / p}}=\infty
$$

then there exists a function $f \in \Lambda(p, \omega)$ which is not a Bloch function.

We remark that Theorem 1.2 is essentially proved in Theorem 1 of [11] (with the con$\operatorname{dition} \lim _{\delta \rightarrow 0} \frac{\omega(\delta)}{\delta^{1 / p}}=\infty$ instead of (1.7)). The arguments used there to prove the result make use of certain sequences associated with $\omega$ which were introduced by Oskolkov in several papers (see e.g. [17], [18], [19] and [20]). In section 2 we shall present a proof of Theorem 2 which is much simpler than that of Theorem 1 of [11]. In particular, we shall not make use of the Oskolkov's sequences.

For the case $p=1$ we can state a stronger result even without conditions on $\phi$. We recall that a function $f$ which is meromorphic in $\Delta$ is a normal function if and only if

$$
\sup _{z \in \Delta}\left(1-|z|^{2}\right) \frac{\left|f^{\prime}(z)\right|}{1+|f(z)|^{2}}<\infty .
$$

We refer to [1] and [21] for the theory of normal functions. For simplicity, let $\mathcal{N}$ denote the set of all holomorphic normal functions in $\Delta$. It is well known that any Bloch function is a normal function, that is, $\mathcal{B} \subset \mathcal{N}$. The second author has proved in [10] the following result.

Theorem D. Let $\phi$ be any positive continuous function defined in $[0,1)$ with $\phi(r) \rightarrow \infty$, as $r \rightarrow 1$. Then, there exists a function $f \in \mathcal{L}(1, \phi)$ which is not a normal function.

We remark that the function $f$ constructed in [10] to prove Theorem $\mathrm{D}$ is of the form $f(z)=F(z) B(z)$ where $B$ is a Blaschke product while the function $F$ is given by a series of analytic functions in $\Delta$ which converges uniformly on every compact subset of $\Delta$. In constructing both $F$ and $B$ we made use of the Oskolkov's sequences.

These results lead to the following improvement of Theorem B.

Theorem 1.3. Let $\phi$ be a non-negative and increasing function defined in $[0,1)$. Then the following conditions are equivalent:

(i) $\mathcal{L}(1, \phi) \subset H^{\infty}$.

(ii) $\mathcal{L}(1, \phi) \subset B M O A$.

(iii) $\mathcal{L}(1, \phi) \subset \mathcal{B}$.

(iv) $\mathcal{L}(1, \phi) \subset \mathcal{N}$.

(v) $\phi$ is bounded.

In Theorem 1.3 the implications (i) $\Rightarrow$ (ii) $\Rightarrow$ (iii) $\Rightarrow$ (iv) follow trivially from the inclusions $H^{\infty} \subset B M O A \subset \mathcal{B} \subset \mathcal{N}$, the implication (iv) $\Rightarrow$ (v) follows from Theorem $\mathrm{D}$ and the implication $(\mathrm{v}) \Rightarrow(\mathrm{i})$ follows from Theorem B.

In view of Theorem $\mathrm{D}$ and Theorem 1.3, it seems natural to expect that, in the conclusion of Theorem 1.2, the condition $f \notin \mathcal{B}$ can be changed to $f$ not normal. This question was already considered in [11] where only a partial result was obtained in this direction [11, Th. 2]. Now we can prove the following much stronger result. 
Theorem 1.4. Let $1 \leq p<\infty$ and let $\omega:[0,1] \rightarrow[0, \infty)$ be a continuous and increasing function with $\omega(0)=0$. Suppose that

$$
\frac{\omega(\delta)}{\delta^{1 / p}} \rightarrow \infty, \quad \text { as } \delta \rightarrow 0
$$

Then there exists a function $f$ analytic in $\Delta$ with

$$
M_{p}\left(r, f^{\prime}\right)=\mathrm{O}\left(\frac{\omega(1-r)}{1-r}\right), \quad \text { as } r \rightarrow 1
$$

which is not a normal function.

We remark that the function $\omega$ of Theorem 1.4 is supposed neither to be a Dini-weight nor to satisfy the condition $b_{1}$. When $\omega$ is required to satisfy these two conditions, we obtain the following extension of Theorem 2 which shows that the condition $\rho^{1 / 2} \in b_{\frac{1}{2 p}}$ is not needed in Theorem 2 of [11].

Theorem 1.5. Let $1 \leq p<\infty$ and let $\omega:[0,1] \rightarrow[0, \infty)$ be a continuous and increasing function with $\omega(0)=0$. Suppose that $\omega$ is a Dini-weight, and $\omega \in b_{1}$. If

$$
\lim _{\delta \rightarrow 0} \frac{\omega(\delta)}{\delta^{1 / p}}=\infty
$$

then there exists a function $f \in \Lambda(p, \omega)$ which is not a normal function.

The function $f$ that we are going to construct to prove Theorem 1.4 will be of the form $f(z)=F(z) B(z)$ where $F$ is a conformal mapping from $\Delta$ onto an appropiate domain $D$ and $B$ is a Blaschke product. The construction of the conformal mapping $F$ will be independent of the Oskolkov's sequences and, in the case $p>1$, the Blaschke product can be taken to be that with zeros at the points $1-2^{-n}$. However, in the case $p=1$ we need to take as $B$ one of the Blaschke products considered in [10] and [11] whose construction depends on the Oskolkov's sequences. Section 3 will be devoted to present the results concerning conformal mappings needed to construct the function $F$ and the proof of Theorem 1.4 will be presented in Section 4 .

Assuming that $\phi(t)=\frac{\omega(1-t)}{1-t}$ is integrable in $(0,1)$ it is simple to see that $\mathcal{L}(p, \phi) \subset H^{p}$ for $p \geq 1$. Upon looking at Theorem 1.1 and Theorem 1.3, it is rather natural to look for conditions on the weight to get the embeddings from $\mathcal{L}(p, \phi)$ into the Hardy spaces $H^{q}$ for $q>p$. Our next results give a complete answer to such a question.

Theorem 1.6. Let $1<p<q<\infty$ and let $\omega$ be a Dini-weight, continuous, non-decreasing with $\omega(0)=0$ and $\omega \in b_{1}$. Then $\Lambda(p, \omega) \subset H^{q}$ if and only if $\omega(t) t^{-\frac{1}{p}} \in L^{q}((0,1))$.

Theorem 1.7. Let $1<q<\infty$ and let $\phi$ be a continuous and non-decreasing function defined in $[0,1)$ such that $\omega(t)=t \phi(1-t)$ is a Dini-weight. Then $\mathcal{L}(1, \phi) \subset H^{q}$ if and only if $\phi \in L^{q}((0,1))$.

The proofs of Theorem 1.2, Theorem 1.6 and Theorem 1.7 will be given in Section 2 .

Let us remark that from now on we shall be using the convention that $C$ will denote a positive constant (which may depend on $\omega, p, B, f, F$ but not on $t, r, \delta$ or $n$ ) and which may be different at each occurrence. 


\section{Proofs of the Theorems for special Weight functions.}

Proof of Theorem 1.2.

Let $\omega$ and $p$ be as in Theorem 1.2. Set

$$
f(z)=\int_{0}^{1} \frac{\omega(t)}{t(1+t-z)^{1 / p}} d t, \quad z \in \Delta .
$$

It is clear that $f$ is holomorphic in $\Delta$. Using Minkowski's integral inequality and the well known fact that $\int_{-\pi}^{\pi} \frac{d \theta}{\left|1-r e^{i \theta}\right|^{\lambda}} \leq \frac{C_{\lambda}}{(1-r)^{\lambda-1}}, 0<r<1, \lambda>1$, we have

$$
\begin{aligned}
M_{p}\left(r, f^{\prime}\right) & =\left(\frac{1}{2 \pi} \int_{-\pi}^{\pi}\left|f^{\prime}\left(r e^{i \theta}\right)\right|^{p} d \theta\right)^{1 / p} \\
& \leq C\left(\frac{1}{2 \pi} \int_{-\pi}^{\pi}\left[\int_{0}^{1} \frac{\omega(t)}{\left|1+t-r e^{i \theta}\right|^{1+\frac{1}{p}}} \frac{d t}{t}\right]^{p} d \theta\right)^{1 / p} \\
& \leq C \int_{0}^{1}\left(\frac{1}{2 \pi} \int_{-\pi}^{\pi}\left[\frac{1}{\left|1+t-r e^{i \theta}\right|^{1+\frac{1}{p}}}\right]^{1 / p} d \theta\right)^{\frac{\omega}{t}(t)} d t \\
& \leq C \int_{0}^{1} \frac{\omega(t)}{t(1+t-r)} d t \\
& =C \int_{0}^{1-r} \frac{\omega(t)}{t(1+t-r)} d t+C \int_{1-r}^{1} \frac{\omega(t)}{t(1+t-r)} d t .
\end{aligned}
$$

Since $\omega$ is a Dini-weight, we obtain

$$
\int_{0}^{1-r} \frac{\omega(t)}{t(1+t-r)} d t \leq \frac{1}{1-r} \int_{0}^{1-r} \frac{\omega(t)}{t} d t \leq C \frac{\omega(1-r)}{1-r} .
$$

On the other hand,

$$
\int_{1-r}^{1} \frac{\omega(t)}{t(1+t-r)} d t=\int_{1-r}^{1} \frac{\omega(t)}{t^{2}} \frac{t}{1+t-r} d t
$$

which, having in mind that $\frac{t}{1+t-r} \leq 1$, if $1-r \leq t<1$, and using that $\omega \in b_{1}$, shows that

$$
\int_{1-r}^{1} \frac{\omega(t)}{t(1+t-r)} d t \leq \int_{1-r}^{1} \frac{\omega(t)}{t^{2}} d t \leq C \frac{\omega(1-r)}{1-r} .
$$

Then (2.1), (2.2) and (2.3) imply $M_{p}\left(r, f^{\prime}\right) \leq C \frac{\omega(1-r)}{1-r}$ and, hence, $f \in \Lambda(p, \omega)$.

Finally, having in mind that $\omega$ is increasing and the trivial inequality $(1+t-r) \leq 3(1-r)$, which holds if $(1-r) \leq t \leq 2(1-r)$, we obtain

$$
f^{\prime}(r) \geq C \int_{1-r}^{2(1-r)} \frac{\omega(t)}{t(1+t-r)^{1+\frac{1}{p}}} d t \geq C \frac{\omega(1-r)}{(1-r)^{1+\frac{1}{p}}} \log 2, \quad \frac{1}{2}<r<1 .
$$


Hence $(1-r)\left|f^{\prime}(r)\right| \geq C \frac{\omega(1-r)}{(1-r)^{1 / p}}$. This and (1.7) clearly imply that $f \notin \mathcal{B}$. This finishes the proof of Theorem 1.2.

Proof of Theorem 1.6.

Let $\omega, p$ and $q$ be as in Theorem 1.6. Let $p^{\prime}$ and $q^{\prime}$ denote the conjugate exponents of $p$ and $q$, respectively, that is, $\frac{1}{p^{\prime}}+\frac{1}{p}=1$ and $\frac{1}{q^{\prime}}+\frac{1}{q}=1$.

First, assume that $\Lambda(p, \omega) \subset H^{q}$. Let $f$ be the function constructed in the proof of Theorem 1.2 , that is

$$
f(z)=\int_{0}^{1} \frac{\omega(t)}{t(1+t-z)^{1 / p}} d t
$$

we have shown that $f \in \Lambda(p, \omega)$. Hence $f \in H^{q}$.

Applying now the Fejér-Riesz inequality (see [7], page 46), we conclude that

$$
\int_{0}^{1}|f(r)|^{q} d r<\infty
$$

On the other hand, having in mind that $\omega$ is increasing, we obtain

$$
\begin{aligned}
\int_{0}^{1}|f(r)|^{q} d r & =\int_{0}^{1}\left(\int_{0}^{1} \frac{\omega(t)}{t(1+t-r)^{1 / p}} d t\right)^{q} d r \\
& =\int_{0}^{1}\left(\int_{0}^{1} \frac{\omega(t)}{t(s+t)^{1 / p}} d t\right)^{q} d s \\
& \geq \int_{0}^{1} \omega(s)^{q}\left(\int_{s}^{1} \frac{d t}{t(s+t)^{\frac{1}{p}}}\right)^{q} d s \\
& \geq C \int_{0}^{1} \omega(s)^{q}\left(\int_{s}^{1} t^{-\left(1+\frac{1}{p}\right)} d t\right)^{q} d s \\
& \geq C \int_{0}^{1} \frac{\omega(s)^{q}}{s^{\frac{q}{p}}} d s .
\end{aligned}
$$

Therefore $\omega(t) t^{-\frac{1}{p}} \in L^{q}((0,1))$.

Conversely, let us assume that $\omega(t) t^{-\frac{1}{p}} \in L^{q}((0,1))$. Using duality (see [4]) it is enough to show that

$$
\int_{0}^{1} \frac{\omega(1-r)}{1-r} M_{p^{\prime}}(f, r) d r \leq C\|f\|_{H^{q^{\prime}}} .
$$

Recall now the following remarkable fact proved by Hardy and Littlewood (see page 87 of $[7])$ :

If $0<p_{1}<p_{2} \leq \infty$ then for any $\lambda \geq p_{1}$ we have

$$
\left(\int_{0}^{1}(1-r)^{\lambda\left(\frac{1}{p_{1}}-\frac{1}{p_{2}}\right)-1} M_{p_{2}}^{\lambda}(f, r) d r\right)^{\frac{1}{\lambda}} \leq C\|f\|_{H^{p_{1}}} .
$$


Now from Hölder's inequality and (2.4) (for $p_{1}=q^{\prime}, p_{2}=p^{\prime}, \lambda=q^{\prime}$ ) we obtain

$$
\begin{aligned}
& \int_{0}^{1} \frac{\omega(1-r)}{1-r} M_{p^{\prime}}(f, r) d r \\
\leq & \left(\int_{0}^{1} \frac{\omega^{q}(1-r)}{(1-r)^{\frac{q}{p}}} d r\right)^{\frac{1}{q}}\left(\int_{0}^{1}(1-r)^{\frac{-q^{\prime}}{p^{\prime}}} M_{p^{\prime}}^{q^{\prime}}(f, r) d r\right)^{\frac{1}{q^{\prime}}} \\
\leq & C\|f\|_{H^{q^{\prime}}} .
\end{aligned}
$$

This finishes the proof of Theorem 1.3.

\section{Proof of Theorem 1.7.}

Let $q, \phi$ and $\omega$ be as in Theorem 1.7.

Assume first that $\mathcal{L}(1, \phi) \subset H^{q}$. Set $F(t)=-\phi(1-t), 0<t \leq 1$. Then $F$ is continuous and increasing. Define

$$
f(z)=\int_{0}^{1} \frac{t d F(t)}{1+t-z}, \quad z \in \Delta,
$$

that is, $f(z)$ is the Lebesgue-Stieltjes integral of $\frac{t}{1+t-z}$ with respect to $F$. We remark that, since $F$ is increasing, $d F$ is a positive measure.

It is clear that $f$ is holomorphic in $\Delta$ and we have

$$
\begin{aligned}
M_{1}\left(r, f^{\prime}\right) & =\frac{1}{2 \pi} \int_{-\pi}^{\pi}\left|f^{\prime}\left(r e^{i \theta}\right)\right| d \theta \\
& \leq \frac{1}{2 \pi} \int_{-\pi}^{\pi} \int_{0}^{1} \frac{t d F(t)}{\left|1+t-r e^{i \theta}\right|^{2}}, d \theta \\
& =\int_{0}^{1} t \frac{1}{2 \pi} \int_{-\pi}^{\pi} \frac{d \theta}{\left|1+t-r e^{i \theta \mid}\right|^{2}} d F(t) \\
& \leq \int_{0}^{1} \frac{t}{1+t-r} d F(t) \\
& \leq \frac{1}{1-r} \int_{0}^{1-r} t d F(t)+\int_{1-r}^{1} d F(t) .
\end{aligned}
$$

Now, integrating by parts and having in mind the definitions of $F$ and $\omega$ and the fact that $\omega$ is a Dini-weight, we obtain

$$
\begin{aligned}
& \int_{0}^{1-r} t d F(t)=\int_{0}^{1-r} \phi(1-t) d t-(1-r) \phi(r) \\
= & \int_{0}^{1-r} \frac{\omega(t)}{t} d t-(1-r) \phi(r) \leq C \omega(1-r)-(1-r) \phi(r) \\
= & C(1-r) \phi(r) .
\end{aligned}
$$

On the other hand,

$$
\int_{1-r}^{1} d F(t)=\phi(r)-\phi(0)
$$


which, with (2.5) and (2.6), gives $M_{1}\left(r, f^{\prime}\right) \leq C \phi(r)$, that is, $f \in \mathcal{L}(1, \phi)$ and, hence $f \in H^{q}$. Observe that, since $\frac{t}{1+t-r} \geq \frac{1}{2}$ if $1-r \leq t<1$,

$$
f(r) \geq C \int_{1-r}^{1} d F(t) \geq C(\phi(r)-\phi(0)) .
$$

Then, again the Fejér-Riesz inequality gives that $\phi \in L^{q}((0,1))$.

Conversely, assume that $\phi \in L^{q}((0,1))$ and take $f(z)=\sum_{n=0}^{\infty} a_{n} z^{n} \in \mathcal{L}(1, \phi)$. Then, for $0<r<1$ and $n \geq 1$,

$$
n r^{n-1}\left|a_{n}\right| \leq C \phi(r)
$$

Therefore,

$$
\left|a_{n}\right| \leq C \frac{1}{n} \phi\left(1-\frac{1}{n}\right)
$$

Consequently, since $\phi$ is increasing,

$$
\begin{aligned}
\sum_{n=1}^{\infty}\left|a_{n}\right|^{q} n^{q-2} & \leq C \sum_{n=1}^{\infty} \frac{\phi^{q}\left(1-\frac{1}{n}\right)}{n(n+1)} \\
& \leq C \sum_{n=1}^{\infty} \int_{1-\frac{1}{n}}^{1-\frac{1}{n+1}} \phi^{q}(t) d t \\
& \leq C \int_{0}^{1} \phi^{q}(t) d t .
\end{aligned}
$$

Consequently, we have that

$$
\sum_{n=1}^{\infty}\left|a_{n}\right|^{q} n^{q-2}<\infty
$$

Then, using Theorem 6.3 in p. 97 of [7], we have that $f \in H^{q}$ if $2<q<\infty$.

To deal with the case $1<q<2$ we shall use duality. Take a polynomial $g$. ¿From (2.4) (for $\lambda=p_{1}=q^{\prime}, p_{2}=\infty$ ) we can write

$$
\left(\int_{0}^{1} M_{\infty}^{q^{\prime}}(r, g) d r\right)^{\frac{1}{q^{\prime}}} \leq C\|g\|_{H^{q^{\prime}}}
$$

Therefore, using (2.7),

$$
\begin{aligned}
\left|\int_{-\pi}^{\pi}\left(f\left(e^{i \theta}\right)-f(0)\right) \bar{g}\left(e^{i \theta}\right) \frac{d \theta}{2 \pi}\right| & =\frac{1}{2}\left|\int_{0}^{1} \int_{-\pi}^{\pi} f^{\prime}\left(r e^{i \theta}\right) \bar{g}\left(r e^{i \theta}\right) e^{i \theta} \frac{d \theta}{2 \pi} d r\right| \\
& \leq \frac{1}{2} \int_{0}^{1} M_{1}\left(r, f^{\prime}\right) M_{\infty}(r, g) d r \\
& \leq C \int_{0}^{1} \phi(r) M_{\infty}(r, g) d r \\
& \leq C\left(\int_{0}^{1} \phi(r)^{q} d r\right)^{\frac{1}{q}}\left(\int_{0}^{1} M_{\infty}^{q^{\prime}}(r, g) d r\right)^{\frac{1}{q^{\prime}}} \leq C\|g\|_{H^{q^{\prime}}} .
\end{aligned}
$$

By duality, this shows that $f \in H^{q}$. This finishes the proof for all values of $q$. 


\section{Some RESUlts on CONFORMAL MAPPings.}

We start recalling a few facts about circularly symmetric functions. A domain $D$ in $\mathbb{C}$ is said to be circularly symmetric if, for every $r$ with $0<r<\infty, D \cap\{|z|=r\}$ is either empty, is the whole circle $|z|=r$, or is a single arc on $|z|=r$ which contains $z=r$ and is symmetric with respect to the real axis.

Let $D$ be a simply connected and circularly symmetric domain in $\mathbb{C}$ with $D \neq \mathbb{C}$ and $0 \in D$ and let $F$ be the conformal mapping from $\Delta$ onto $D$ with $F(0)=0$ and $F^{\prime}(0)>0$. Then (see [14] or the Corollary in p. 154 of [2]) it is known that:

(a) $M_{\infty}(r, F)=F(r), 0<r<1$.

(b) For every $r$ with $0<r<1,\left|F\left(r e^{i \theta}\right)\right|$ is a decreasing function of $\theta$ in $[0, \pi]$.

We shall also be interested on some results about starlike functions. Let $D \neq \mathbb{C}$ be a domain in $\mathbb{C}$ with $0 \in D$ which is starlike with respect to the origin and let $F$ be the conformal mapping from $\Delta$ onto $D$ with $F(0)=0, F^{\prime}(0)>0$. Then (see p. 43 of [21] or p. 41 of [8])

(c) For every $r$ with $0<r<1, \arg F\left(r e^{i \theta}\right)$ is an increasing function of $\theta$ in $[0,2 \pi]$.

We have the following result.

Theorem 3.1. Let $D$ be a domain in $\mathbb{C}$ with $0 \in D$ and $D \neq \mathbb{C}$. Suppose that $D$ is both circularly symmetric and starlike with respect to 0 . Let $F$ be the conformal mapping from $\Delta$ onto $D$ with $F(0)=0$ and $F^{\prime}(0)>0$. Then there exists a positive constant $C$ such that

$$
2 \pi r M_{1}\left(r, F^{\prime}\right) \leq C M_{\infty}(r, F), \quad 0<r<1 .
$$

Remark 1. Since $2 \pi r M_{1}\left(r, F^{\prime}\right)$ represents the length of the curve $F\left(r e^{i \theta}\right), 0 \leq \theta \leq 2 \pi$, the inequality $M_{\infty}(r, F) \leq 2 \pi r M_{1}\left(r, F^{\prime}\right)$ is trivial.

Remark 2. The conclusion of Theorem 3.1 is not true if we only assume that $D$ is starlike with respect to the origin. Indeed, Hayman proved in [13] that there exists a domain $D$ in $\mathbb{C}$ which is both bounded and starlike with respect to the origin such that if $F$ is the conformal mapping from $\Delta$ onto $D$ with $F(0)=0$ and $F^{\prime}(0)>0$ then

$$
\limsup _{r \rightarrow 1} \frac{M_{1}\left(r, F^{\prime}\right)}{\log \frac{1}{1-r}}>0 .
$$

For this $F$ we have that $F \in H^{\infty}$ and (3.2) shows that $F$ does not satisfy (3.1).

Proof of Theorem 3.1. Given $0<r<1$ let $C_{r}$ denote the image under $F$ of the circle $\{|z|=r\}$, that is, $C_{r}=\left\{F\left(r e^{i \theta}\right), 0 \leq \theta \leq 2 \pi\right\}$. Since $F$ is conformal, $2 \pi r M_{1}\left(r, F^{\prime}\right)$ represents the length of the curve $C_{r}$. Now, since $D$ is symmetric with respect to the real axis,

$$
\text { length }\left(C_{r}\right)=2 \text { length }\left(C_{r}^{+}\right)
$$

where, $C_{r}^{+}=\left\{F\left(r e^{i \theta}\right), 0 \leq \theta \leq \pi\right\}$.

Hence, let $0<r<1$ and let $0=\theta_{0}<\theta_{1}<\cdots<\theta_{n}=\pi$ be any partition of $[0, \pi]$. Denote

$$
F\left(r e^{i \theta_{j}}\right)=s_{j} e^{i \varphi_{j}}, \quad j=0,1, \ldots, n
$$


Since $D$ is circularly symmetric, using (b), we see that $s_{0} \geq s_{1} \geq \cdots \geq s_{n}$. Also, it is clear that $0=\varphi_{0} \leq \varphi \leq \varphi_{n}=\pi$ for all $\varphi \in[0, \pi]$. Then, since $D$ is starlike, using (c), we obtain that $0=\varphi_{0} \leq \varphi_{1} \leq \cdots \leq \varphi_{n}=\pi$.

Then

$$
\begin{aligned}
\sum_{j=1}^{n}\left|F\left(r e^{i \theta_{j}}\right)-F\left(r e^{i \theta_{j-1}}\right)\right| & =\sum_{j=1}^{n}\left|s_{j} e^{i \varphi_{j}}-s_{j-1} e^{i \varphi_{j-1}}\right| \\
& \leq \sum_{j=1}^{n}\left(\left|s_{j} e^{i \varphi_{j}}-s_{j-1} e^{i \varphi_{j}}\right|+\left|s_{j-1} e^{i \varphi_{j}}-s_{j-1} e^{i \varphi_{j-1}}\right|\right) \\
& =\sum_{j=1}^{n}\left(\left(s_{j-1}-s_{j}\right)+s_{j-1}\left|e^{i \varphi_{j}}-e^{i \varphi_{j-1}}\right|\right) \\
& \leq s_{0}+C s_{0} \sum_{j=1}^{n}\left(\varphi_{j}-\varphi_{j-1}\right)=s_{0}+C s_{0} \pi \\
& =(1+C \pi) F(r)=C F(r)=C M_{\infty}(r, F) .
\end{aligned}
$$

Since the partition is arbitrary, this shows that length $\left(C_{r}^{+}\right) \leq C M_{\infty}(r, F)$ and, hence, length $\left(C_{r}\right) \leq C M_{\infty}(r, F)$ which is equivalent to $(3.1)$.

The functions $F$ mentioned in Section 1 which will be used to prove Theorem 1.4 are constructed in Theorem 3.2.

Theorem 3.2. Let $\phi:[0,1) \rightarrow[0, \infty)$ be a function with $\phi(r) \rightarrow \infty$ as $r \rightarrow 1$. Then there exists a domain $D$ in $\mathbb{C}$ with $0 \in D \neq \mathbb{C}$ which is both circularly symmetric and starlike with respect to 0 such that if $F$ is the conformal mapping from $\Delta$ onto $D$ with $F(0)=0$ and $F^{\prime}(0)>0$ then

$$
\begin{gathered}
M_{\infty}(r, F)=F(r) \leq \phi(r), \quad \text { for all } r \text { sufficiently close to } 1, \\
M_{1}\left(r, F^{\prime}\right) \leq \phi(r), \quad \text { for all } r \text { sufficiently close to } 1,
\end{gathered}
$$

and

$$
F(r) \rightarrow \infty, \quad \text { as } r \rightarrow 1 .
$$

Proof of Theorem 3.2. First let us notice that we may assume without loss of generality that $\phi$ is increasing. Otherwise consider $\phi_{1}$, the greatest increasing minorant of $\phi$, defined by

$$
\phi_{1}(r)=\inf _{r \leq s<1} \phi(s), \quad 0 \leq r<1 .
$$

Then it is clear that $\phi_{1} \leq \phi$ and that $\phi_{1}(r) \rightarrow \infty$, as $r \rightarrow 1$. 
Hence we shall assume that $\phi$ is increasing. Let $\left\{r_{n}\right\}_{n=1}^{\infty}$ be a sequence of real numbers with $0<r_{1}<r_{2}<\cdots<1$, such that $\phi\left(r_{n}\right)>n$ for all $n$. Then it is clear that $r_{n} \rightarrow 1$, as $n \rightarrow \infty$.

We define the domain

$$
D=\Delta \cup\left(\bigcup_{n=1}^{\infty}\left\{z:|\operatorname{Im} z|<\gamma_{n}, \operatorname{Re} z>0, n \leq|z|<n+1\right\}\right)
$$

where $\left\{\gamma_{n}\right\}_{n=1}^{\infty}$ is a decreasing sequence of real numbers with $0<\gamma_{n}<1$ to be specified below.

Define $D_{0}=\Delta$,

$$
\begin{aligned}
D_{n} & =D_{n-1} \cup\left\{z:|\operatorname{Im} z|<\gamma_{n}, \operatorname{Re} z>0, n \leq|z|<n+1\right\} \\
& =\Delta \cup\left(\bigcup_{j=1}^{n}\left\{z:|\operatorname{Im} z|<\gamma_{j}, \operatorname{Re} z>0, j \leq|z|<j+1\right\}\right), \quad n \geq 1 .
\end{aligned}
$$

We also define

$$
G_{n}=D_{n-1} \cup\left\{z:|\operatorname{Im} z|<\gamma_{n}, \operatorname{Re} z>0\right\}, \quad n \geq 1
$$

For every $n \geq 1$ let $g_{n}$ be the conformal mapping from $\Delta$ onto $G_{n}$ with $g_{n}(0)=0$ and $g_{n}^{\prime}(0)>0$ and let $f_{n}$ be the conformal mapping from $\Delta$ onto $D_{n}$ with $f_{n}(0)=0$ and $f_{n}^{\prime}(0)>0$. By the Carathèodory kernel theorem (see [8, p. 78] or [21, p. 29]), we can choose $\gamma_{1}$ with $0<\gamma_{1}<1$ so small that

$$
M_{\infty}\left(r_{2}, g_{1}\right) \leq \phi\left(r_{1}\right)
$$

Suppose that $\gamma_{1}>\gamma_{2}>\cdots>\gamma_{n-1}$ have been chosen so that

$$
M_{\infty}\left(r_{j+1}, g_{j}\right) \leq \phi\left(r_{j}\right), \quad 1 \leq j \leq n-1
$$

Again, using the Carathèodory kernel theorem, we see that we can choose $\gamma_{n}$ with $0<$ $\gamma_{n}<\gamma_{n-1}$ such that

$$
M_{\infty}\left(r_{n+1}, g_{n}\right) \leq \phi\left(r_{n}\right)
$$

Notice that, for $j \leq n, f_{n}$ is subordinate to $g_{j}$ and hence (3.10) and (3.11) (see Chapter 2 of [21]) show that

$$
M_{\infty}\left(r_{j+1}, f_{n}\right) \leq \phi\left(r_{j}\right), \quad 1 \leq j \leq n, \quad n \geq 1
$$

Notice that $D=\cup_{n=1}^{\infty} D_{n}$. Then $D$ is a domain which is both circularly symmetric and starlike with respect to the origin. Also, it is clear that $D$ is the kernel of the sequence $\left\{D_{n}\right\}$. Hence, by the Carathèodory kernel theorem, if $F$ is the conformal mapping from 
$\Delta$ onto $D$ with $F(0)=0$ and $F^{\prime}(0)>0$, we have that $f_{n} \rightarrow F$, as $n \rightarrow \infty$ uniformly on every compact subset of $\Delta$. Then, using (3.12), se obtain

$$
M_{\infty}\left(r_{j+1}, F\right) \leq \phi\left(r_{j}\right), \quad \text { for all } j \geq 1 .
$$

Now, if $r_{1}<r<1$, there exists $j \geq 1$ such that $r_{j}<r \leq r_{j+1}$. Then, since $M_{\infty}(r, F)$ and $\phi(r)$ are increasing functions of $r$,

$$
M_{\infty}(r, F) \leq M_{\infty}\left(r_{j+1}, F\right) \leq \phi\left(r_{j}\right) \leq \phi(r)
$$

This proves (3.3). Now (3.4) follows from (3.3) and Theorem 3.1.

To prove (3.5) just notice that, since $D$ is circularly symmetric and $F^{\prime}(0)>0$,

$$
F(r)=|F(r)|=M_{\infty}(r, F), \quad 0<r<1,
$$

and that, since $D$ is not bounded, $M_{\infty}(r, F) \rightarrow \infty$, as $r \rightarrow 1$. This finishes the proof.

\section{Proof of TheOrem 1.4.}

We start with the following simple result on a particular Blaschke product.

Proposition 4.1. Let

$$
a_{n}=1-2^{-n}, \quad n \geq 1,
$$

and let $B$ be the Blaschke product whose zeros are $\left\{a_{n}\right\}_{n=1}^{\infty}$. Then, for $1<p<\infty$,

$$
M_{p}\left(r, B^{\prime}\right)=\mathrm{O}\left(\frac{1}{(1-r)^{1-\frac{1}{p}}}\right), \quad \text { as } r \rightarrow 1 .
$$

Proof of Proposition 4.1. We have $B(z)=\prod_{n=1}^{\infty} \frac{a_{n}-z}{1-a_{n} z}$. Hence,

$$
\left|B^{\prime}(z)\right| \leq \sum_{n=1}^{\infty} \frac{1-\left|a_{n}\right|^{2}}{\left|1-a_{n} z\right|^{2}}\left|\prod_{j \neq n} \frac{a_{j}-z}{1-a_{j} z}\right| \leq \sum_{n=1}^{\infty} \frac{1-a_{n}^{2}}{\left|1-a_{n} z\right|^{2}} \leq 2 \sum_{n=1}^{\infty} \frac{1-a_{n}}{\left|1-a_{n} z\right|^{2}} .
$$

Therefore,

$$
M_{p}\left(r, B^{\prime}\right) \leq C \sum_{n=1}^{\infty}\left(1-a_{n}\right)\left(\frac{1}{2 \pi} \int_{0}^{2 \pi} \frac{d \theta}{\left|1-a_{n} r e^{i \theta}\right|^{2 p}}\right)^{1 / p} \leq C \sum_{n=1}^{\infty} \frac{1-a_{n}}{\left(1-a_{n} r\right)^{2-\frac{1}{p}}} .
$$

The inequality $2\left(1-a_{n} r\right) \geq 1-a_{n}+1-r$ and the definition of $a_{n}$ now imply that

$$
\begin{aligned}
M_{p}\left(r, B^{\prime}\right) & \leq C \sum_{n=1}^{\infty} \frac{2^{-n}}{\left(2^{-n}+1-r\right)^{2-\frac{1}{p}}} \\
& \leq C \sum_{n=0}^{\infty} \int_{2^{-(n+1)}}^{2^{-n}} \frac{d t}{(t+1-r)^{2-\frac{1}{p}}} \\
& =C \int_{0}^{1} \frac{d t}{(t+1-r)^{2-\frac{1}{p}}} \\
& \leq \frac{C}{(1-r)^{1-\frac{1}{p}}} .
\end{aligned}
$$


This proves (4.1).

Proof of Theorem 1.4. We shall consider first the case $1<p<\infty$. Set

$$
\phi(r)=\frac{\omega(1-r)}{(1-r)^{1 / p}}, \quad 0<r<1 .
$$

Then $\phi$ satisfies the conditions of Theorem 3.2. Hence, let $F$ be the function constructed in Theorem 3.2 with this $\phi$. Then $F$ satisfies (3.3), (3.4) and (3.5). Using (3.3) and arguing as in the proof of Theorem 5.5 of [7], we obtain

$$
M_{\infty}\left(r, F^{\prime}\right) \leq C \frac{M_{\infty}\left(\frac{1+r}{2}, F\right)}{1-r}
$$

which, with (3.4), implies

$$
\begin{aligned}
M_{p}\left(r, F^{\prime}\right)^{p} & \leq M_{1}\left(r, F^{\prime}\right) M_{\infty}\left(r, F^{\prime}\right)^{p-1} \\
& \leq C \phi(r) \frac{\phi\left(\frac{1+r}{2}\right)^{p-1}}{(1-r)^{p-1}} .
\end{aligned}
$$

But, since $\omega$ is increasing,

$$
\phi\left(\frac{1+r}{2}\right)=\frac{\omega\left(\frac{1-r}{2}\right)}{\left(\frac{1-r}{2}\right)^{1 / p}} \leq C \phi(r) .
$$

Hence, we have

$$
M_{p}\left(r, F^{\prime}\right)^{p} \leq C \frac{\phi(r)^{p}}{(1-r)^{p-1}}
$$

or, equivalently,

$$
M_{p}\left(r, F^{\prime}\right) \leq C \frac{\phi(r)}{(1-r)^{1-\frac{1}{p}}}=C \frac{\omega(1-r)}{1-r} .
$$

Now, we let $B$ be the Blaschke product considered in Proposition 4.1, that is, that with zeros at the points $a_{n}=1-2^{-n}, n \geq 1$. Finally, we set $f(z)=F(z) B(z), z \in \Delta$. Since $\left\{a_{n}\right\}$ is uniformly separated, using (3.5) we dedude that $f$ is not a normal function arguing just as in the proofs of Theorem 1 of [10] or in that of Theorem 2 of [11]. Furthermore,

$$
M_{p}\left(r, f^{\prime}\right) \leq M_{p}\left(r, F^{\prime}\right) M_{\infty}(r, B)+M_{\infty}(r, F) M_{p}\left(r, B^{\prime}\right)
$$

Then, (4.2), the fact that $B \in H^{\infty},(3.3)$, Proposition 4.1 and the definition of $\phi$ imply

$$
M_{p}\left(r, f^{\prime}\right)=\mathrm{O}\left(\frac{\omega(1-r)}{1-r}\right), \quad \text { as } r \rightarrow 1
$$

This finishes the proof in the case $p>1$. 
In the case $p=1$ we take $F$ as in the case $p>1$ but taking

$$
\phi(r)=\left(\frac{\omega(1-r)}{1-r}\right)^{1 / 2},
$$

and we take as $B$ an interpolating Blaschke product $B$ with positive zeros having the property that

$$
M_{1}\left(r, B^{\prime}\right)=\mathrm{O}(\phi(r)), \quad \text { as } r \rightarrow 1 .
$$

Such a Blaschke product is constructed in Theorem 3 of [11]. We omit the details.

Remark 3. It is natural to expect that Theorem 1.4 remains true with (1.7) in the place of (1.9). However, our proof does not yield this since it uses that the function $F$ constructed there satisfies $M_{\infty}(r, F)=\mathrm{O}\left(\frac{\omega(1-r)}{(1-r)^{1 / p}}\right)$, as $r \rightarrow 1$.

\section{ReFERENCES}

[1] J. M. Anderson, J. Clunie and Ch. Pommerenke, On Bloch functions and normal functions, J. Reine Angew. Math. 270 (1974), 12-37.

[2] A. Baernstein, Integral means, univalent functions and circular symmetrization, Acta Math. 133 (1974), 139-169.

[3] A. Baernstein, Analytic functions of bounded mean oscillation, Aspects of Contemporary Complex Analysis (D. Brannan and J. Clunie, eds.), Academic Press, 1980, pp. 3-36.

[4] O. Blasco and G. Soares de Souza, Spaces of analytic functions on the disc where the growth of $M_{p}(F, r)$ depends on a weight, J. Math. Anal. Appl. 147 no. 2 (1990), 580-598.

[5] P. Bourdon, J. Shapiro and W. Sledd, Fourier series, mean Lipschitz spaces and bounded mean oscillation, Analysis at Urbana 1, Proceedings of the Special Year in Modern Analysis at the University of Illinois, 1986-87 (E. R. Berkson, N. T. Peck and J. Uhl, eds.), London Math. Soc. Lecture note series 137, Cambridge Univ. Press, 1989, pp. 81-110.

[6] J. A. Cima and K. E. Petersen, Some analytic functions whose boundary values have bounded mean oscillation, Math. Z. 147 (1976), 237-347.

[7] P. L. Duren, Theory of $H^{p}$ spaces, Academic Press (New York), 1970.

[8] P. L. Duren, Univalent functions, Springer-Verlag, New York, 1983.

[9] J. B. Garnett, Bounded analytic functions, Academic Press (New York), 1981.

[10] D. Girela, On a theorem of Privalov and normal functions, Proc. Amer. Math. Soc. 125 no. 2 (1997), 433-442.

[11] D. Girela, Mean Lipschitz spaces and bounded mean oscillation, to appear in Illinois J. Math..

[12] G. H. Hardy and J. E. Littlewood, Some properties of fractional integrals, II, Math. Z., 34 (1932), 403-439.

[13] W. K. Hayman, On functions with positive real part, J. London Math. Soc. 36 (1961), 35-48.

[14] J. A. Jenkins, On circularly symmetric functions, Proc. Amer. Math. Soc. 6 (1955), 620-624.

[15] O. Lehto and K. I. Virtanen, Boundary behaviour and normal meromorphic functions, Acta Math. 97 (1957), 47-65.

[16] G. D. Levshina, Coefficient multipliers of Lipschitz functions, Mat. Zametki, vol. 52 no. 5 (1992), pp. 68-77 (Russian); English transl. in Math. Notes, vol. 52 no. 5-6 (1992), pp. 1124-1130 (1993).

[17] K. I. Oskolkov, An estimate of the rate of approximation of a continuous function and its conjugate by Fourier sums on a set of total measure, Izv. Acad. Nauk SSSR Ser. Mat. 38 no. 6 (1974), 1393-1407 (Russian); English transl. in Math. USSR Izv. 8 no. 6 (1974), 1372-1386 (1976).

[18] K. I. Oskolkov, Uniform modulus of continuity of summable functions on sets of positive measure, Dokl. Akad. Nauk SSSR 229 no. 2 (1976), 304-306 (Russian); English transl. in Sov. Math. Dokl. 17 no. 4 (1976), 1028-1030 (1977). 
[19] K. I. Oskolkov, Approximation properties of summable functions on sets of full measure, Mat. Sbornik, n. Ser. 103(145) (1977), 563-589 (Russian); English transl. in Math. USSR Sbornik 32 no. 4 (1977), 489-514 (1978).

[20] K. I. Oskolkov, On Luzin's C-property for a conjugate function, Trudy Mat. Inst. Stelklova 164 (1983), 124-135 (Russian); English transl. in Proc. Steklov Inst. Math. 164 (1985), 141-153.

[21] Ch. Pommerenke, Univalent Functions, Vandenhoeck und Ruprecht, Göttingen, 1975.

O. Blasco, Departamento de Análisis Matemático, Universidad de Valencia, 46100 Burjasot, SPAIN

E-mail: oblasco@uv.es

D. Girela, Departamento de Análisis Matemático, Facultad de Ciencias, Universidad de Málaga, 29071 Málaga, SPAin

E-mail: girela@anamat.cie.uma.es

M.A. Márquez, Departamento de Análisis Matemático, Facultad de Ciencias, Universidad DE Málaga, 29071 Málaga, Spain

E-mail: marquez@anamat.cie.uma.es 